\title{
Sensitivity of PARASOL multi-angle photopolarimetric aerosol retrievals to cloud contamination
}

\author{
F. A. Stap ${ }^{1,2}$, O. P. Hasekamp ${ }^{1}$, and T. Röckmann ${ }^{2}$ \\ ${ }^{1}$ SRON Netherlands Institute for Space Research, Utrecht, the Netherlands \\ ${ }^{2}$ Institute for Marine and Atmospheric Research (IMAU), Utrecht University, Utrecht, the Netherlands \\ Correspondence to: F. A. Stap (f.a.stap@sron.nl)
}

Received: 26 August 2014 - Published in Atmos. Meas. Tech. Discuss.: 23 October 2014

Revised: 26 February 2015 - Accepted: 27 February 2015 - Published: 17 March 2015

\begin{abstract}
An important problem in satellite remote sensing of aerosols is related to the need to perform an adequate cloud screening. If a cloud screening is applied that is not strict enough, the ground scene has the probability of residual cloud cover which causes large errors on the retrieved aerosol parameters. On the other hand, if the cloudscreening procedure is too strict, too many clear sky cases, especially near-cloud scenes, will falsely be flagged cloudy. The detrimental effects of cloud contamination as well as the importance of aerosol cloud interactions that can be studied in these near-cloud scenes call for new approaches to cloud screening. Multi-angle multi-wavelength photopolarimetric measurements have a unique capability to distinguish between scattering by (liquid) cloud droplets and aerosol particles. In this paper the sensitivity of aerosol retrievals from multi-angle photopolarimetric measurements to cloud contamination is investigated and the ability to intrinsically filter the cloud-contaminated scenes based on a goodnessof-fit criteria is evaluated. Hereto, an aerosol retrieval algorithm is applied to a partially clouded over-ocean synthetic data set as well as non-cloud-screened over-ocean POLDER3/PARASOL observations. It is found that a goodness-of-fit filter, together with a filter on the coarse mode refractive in$\operatorname{dex}\left(m_{\mathrm{r}}^{\text {coarse }}>1.335\right)$ and a cirrus screening, adequately rejects the cloud-contaminated scenes. No bias or larger SD are found in the retrieved parameters for this intrinsic cloud filter compared to the parameters retrieved in a priori cloudscreened data set (using MODIS/AQUA cloud masks) of PARASOL observations. Moreover, less high-aerosol load scenes are misinterpreted as cloud contaminated. The retrieved aerosol optical thickness, single scattering albedo and Ångström exponent show good agreement with AERONET
\end{abstract}

observations. Furthermore, the synthetic retrievals give confidence in the ability of the algorithm to correctly retrieve the micro-physical aerosol parameters.

\section{Introduction}

Aerosol plays a complex role in our atmosphere that results in a net negative radiative forcing. The uncertainty on the strength of this aerosol forcing is the largest contribution to the uncertainty on total radiative forcing estimates (IPCC, 2014) and complicates future climate predictions (Hansen et al., 2011). To reduce the large uncertainty of the aerosol effects on cloud formation and climate, accurate satellite measurements of aerosol optical properties (optical thickness, single scattering albedo (SSA), phase function) and micro-physical properties (size distribution, refractive index, shape) are essential. Knowledge of the optical properties is needed to estimate the forcing due to the direct effect and semi-direct effects (the latter depends on the absorption by aerosols). The refractive index, which is a proxy for aerosol chemical composition, and the aerosol size distribution are the most important characteristics to distinguish man-made aerosols from natural aerosols. Furthermore, the capability of aerosols to act as cloud condensation nuclei (CCN) depends on the number of aerosol particles that in "dry" form (i.e., without water uptake) have a radius that is larger than about $0.05 \mu \mathrm{m}$ (Rosenfeld, 2006). At high relative humidity, however, aerosols often grow by absorbing water. The aerosol refractive index strongly depends on the water uptake by the aerosols and therefore this quantity can be used to translate the measured size distribution of hydrated particles 
to the corresponding size distribution of dry particles (Schuster et al., 2009), which is needed to determine the number of potential CCN.

Satellite instruments that perform multi-angle photopolarimetric measurements have the capability to provide the aerosol properties mentioned above. This has been demonstrated by theoretical studies (Mishchenko and Travis, 1997; Hasekamp and Landgraf, 2007; Kokhanovsky et al., 2010; Knobelspiesse et al., 2012; Ottaviani et al., 2013) as well as by case studies using airborne measurements (Chowdhary et al., 2005; Waquet et al., 2009a). The only satellite instruments that performed multi-angle photopolarimetric measurements were the POLDER (Polarization and Directionality of the Earth's Reflectances) instruments (Deschamps et al., 1994), of which the recently decommissioned POLDER-3 on board the PARASOL (Polarization \& Anisotropy of Reflectances for Atmospheric Sciences coupled with Observations from a Lidar) micro-satellite provided more than 8 years of data.

The retrieval algorithms used for the operational aerosol data products of PARASOL do not yet make full use of the information contained in the measurements. However, more recent studies (Dubovik et al., 2011; Hasekamp et al., 2011) do fully exploit the information of PARASOL measurements and make the retrieval of detailed aerosol properties like size distribution, refractive index and single scattering albedo possible at a global scale.

A complication in satellite remote sensing of aerosols arises from the need to perform an adequate cloud screening. If cloud screening is not applied strictly enough, residual cloud cover can cause large errors on the retrieved aerosol parameters. The downside of performing a strict cloud screening is that clear sky cases may be falsely flagged cloudy, which results in data loss for areas with hydrated aerosols, the so-called Twilight zone (Koren et al., 2007), and areas with high aerosol loading. The problems noted above become particularly relevant in regions close to clouds, where aerosol measurements are important to understand the effect of aerosols on cloud formation.

Multi-angle multi-wavelength measurements of intensity as well as polarization are particularly suitable to distinguish between scattering due to cloud and aerosol particles (Waquet et al., 2009b, 2013; Hasekamp, 2010; Knobelspiesse et al., 2011). One of the benefits of this type of measurement is the observation of a distinct polarization feature at a scattering angle of $14^{\circ}$, known as the cloud bow (see e.g., Hansen and Travis, 1974). As an example, Fig. 1 shows a PARASOL observation of a partially clouded scene together with the best fit of the retrieval algorithm, which is unable to reproduce the cloud-bow feature.

The aim of this paper is to investigate the sensitivity of aerosol retrievals from multi-angle multi-wavelength measurements of intensity as well as polarization to cloud contamination. For this purpose, aerosol retrievals from synthetic measurements and measurements from PARASOL
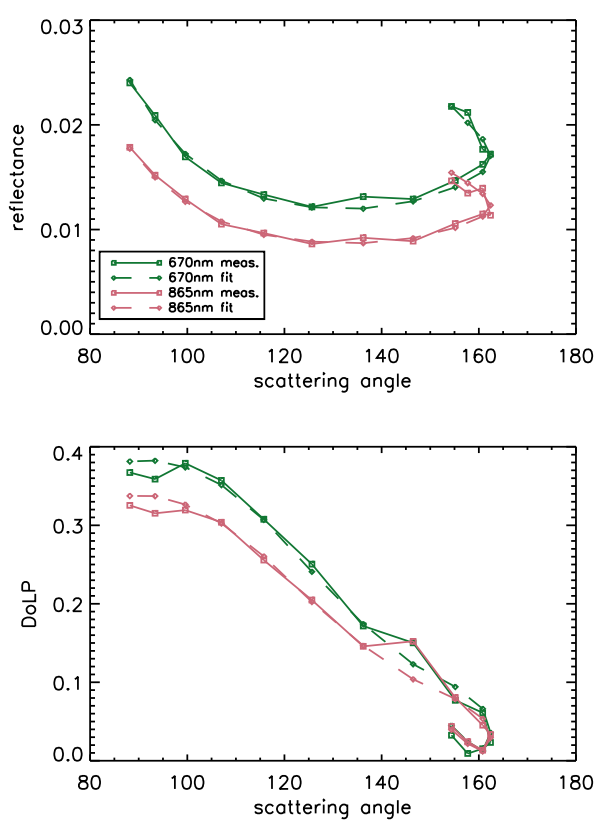

Figure 1. A POLDER-3/PARASOL observation of a scene with $\sim 20 \%$ cover. The 670 and $865 \mathrm{~nm}$ bands are shown in, respectively, green and red. The dashed lines show the best fit to this observation (assuming clear sky conditions). The fit has a high $\chi^{2}$ value $(\sim 13.7)$ mostly due to the discrepancy near the cloud-bow, which is visible in the degree of linear polarization at a scattering angle of $\sim 140^{\circ}$. The measurement errors are of the size of the symbols.

are evaluated. For the latter case, cloud measurements taken from the MODIS-AQUA satellite instrument are colocated with PARASOL observations. The performance of the aerosol retrieval algorithm is evaluated for different cloud-screening algorithms - in particular the case where, instead of a priori cloud screening, an a posteriori screening based on the goodness of fit is applied.

In Sect. 2 the PARASOL and MODIS observations and data sets are described. In Sect. 3 a summary of the inversion method is given. Then, in Sect. 4, a data set of synthetic partially clouded scenes is presented and the performance of the algorithm on this data set is analyzed. In Sect. 5 the algorithm is applied to real, partially clouded observations. The performance is evaluated by comparison to ground-based observations of several AERONET stations (Holben et al., 2001). In Sect. 6, the results are summarized and conclusions are drawn.

\section{Observations}

\subsection{PARASOL}

The recently decommissioned PARASOL satellite was launched in 2004 and flew as part of the NASA A-train for a little less than 5 years, during which it collected aerosol and 
Table 1. A selection of the near-ocean AERONET stations that have been used in the validation.

\begin{tabular}{lrr}
\hline Station & Latitude $\left[^{\circ}\right]$ & Longitude $\left[^{\circ}\right]$ \\
\hline Muscat & 23.61 & 58.44 \\
Anmyon & 36.54 & 126.33 \\
FORTH Crete & 35.33 & 25.28 \\
Gosan SNU & 33.29 & 126.16 \\
Guam & 13.43 & 144.80 \\
Midway Island & 28.21 & -177.38 \\
Shirahama & 33.69 & 135.36 \\
Trelew & -43.25 & -65.31 \\
Trinidad Head & 41.05 & -124.15 \\
Sevastopol & 44.62 & 33.52 \\
\hline
\end{tabular}

cloud observations in synthesis with MODIS/AQUA (multispectral imager), CALIPSO (lidar) and CLOUDSAT. It measured the intensity in nine spectral bands ranging from 443 to $1020 \mathrm{~nm}$ at up to 16 viewing angles. Additionally, the linear polarization was measured in the 490, 670 and $865 \mathrm{~nm}$ bands. The level 1 (non-cloud-screened) observations are available on a sinusoidally projected grid of $\sim 6.2 \mathrm{~km} \times 6.2 \mathrm{~km}$ pixels, named the full-resolution (FR) grid. This data set is processed into a non-cloud-screened medium-resolution (MR) data set of $\sim 19 \mathrm{~km} \times 19 \mathrm{~km}$ pixels for our analysis.

The selection of PARASOL observations that are used in the analysis are comprised of scenes above ocean surfaces that are obtained during the year 2006 and are in the vicinity of one of the AERONET stations listed in Table 1. The latter criterion allows for the validation of the retrieved optical and micro-physical aerosol properties, whenever AERONET observations are available.

\subsection{MODIS cloud product}

Information on cloud cover and cloud properties, at a resolution higher than PARASOL, is obtained by the colocation of both the MODIS/AQUA cloud mask (MYD35, collection 005) (Ackerman et al., 1998) and cloud product (MYD06_L2, collection 005) on the sinusoidally projected PARASOL coordinate grid. The nadir pixel size of the cloud mask and cirrus flag is $\sim 1 \mathrm{~km} \times 1 \mathrm{~km}$. The MODIS geolocations in the MYD06_L2 are provided on $5 \mathrm{~km} \times 5 \mathrm{~km}$ pixel resolution but have been interpolated to the $1 \mathrm{~km} \times 1 \mathrm{~km}$ grid. This provides $32 \pm 5$ and $291 \pm 40$ pixels of roughly $1 \mathrm{~km} \times 1 \mathrm{~km}$ resolution cloud information for, respectively, the FR and MR PARASOL ground pixels. For consistent treatment of the cloud fractions the MODIS observations in this study are restricted to sensor zenith angles lower than $40^{\circ}$. Note that this last criteria excludes the use of the full width of the PARASOL swath.

The MODIS cloud mask has four different flags: confidently clear, probably clear, probably cloudy and confidently cloudy. From this mask two different cloud fractions are derived per PARASOL ground pixel: (i) a conservative ("strict") cloud fraction that counts all pixels that are not "confidently clear" as cloud pixels and (ii) a "loose" cloud fraction which counts both the "probably cloudy" and "confidently cloudy" flags as cloudy pixels (but not the "probably clear"). Furthermore, a cirrus fraction was derived from the fraction of pixels for which cirrus was detected as conveyed via the cirrus reflectance flag (Gao et al., 2002). The MODIS cirrus detection algorithm uses observations from the 0.66 and $1.38 \mu \mathrm{m}$ bands.

\section{Inversion method}

This study uses the retrieval algorithm described in detail by Hasekamp et al. (2011). The retrieval approach is based on iterative fitting of a linearised vector radiative transfer (RT) model, developed at SRON Netherlands Institute for Space Research (Hasekamp and Landgraf, 2002, 2005), to the multi-angle photopolarimetric measurements. The aerosol retrieval algorithm explicitly retrieves the microphysical aerosol properties corresponding to a bi-modal aerosol model. Here, each mode is separately described by an effective radius and width, complex refractive indices, the column number concentration and the fraction of spherical particles (only for the coarse mode aerosol). Additionally, four ocean parameters (wind speed in two directions, Chlorophyll $a$ concentration and white cap fraction) are retrieved simultaneously with the aerosol parameters.

Since the paper of Hasekamp et al. (2011) a number of improvements have been included in the algorithm: (i) the coarse mode is now described by a mixture of spheroids and spheres using the pre-calculated optical properties by Dubovik et al. (2006). The fraction of spherical particles in the coarse mode is included as an additional fit parameter. (ii) The algorithm now uses four wavelength bands, namely $490,670,865$ and $1020 \mathrm{~nm}$, while only two wavelength bands ( 490 and $670 \mathrm{~nm}$ ) were used by Hasekamp et al. (2011). (iii) The measurement vector contains intensity and degree of linear polarization (DoLP) instead of intensity and Stokes fractions $q=Q / I$ and $u=U / I$ as in Hasekamp et al. (2011).

\section{Synthetic retrievals}

\subsection{Partially clouded data set}

A variety of clear sky and partial liquid cloud cover PARASOL observations are simulated to study the sensitivity of the aerosol retrieval algorithm to liquid cloud contamination and the effect of liquid cloud contamination on the retrieved optical and aerosol parameters. The synthetic measurements are created using the linearized vector RT model described by Hasekamp and Landgraf $(2002,2005)$. Three aerosol modes are considered in these calculations. The first two are used 
Table 2. The ranges of the aerosol parameters used to create the synthetic data set.

\begin{tabular}{ll}
\hline Fine mode & Coarse mode \\
\hline $0.04 \leq R_{\text {eff }} \leq 0.5$ & $0.8 \leq R_{\text {eff }} \leq 3.0$ \\
$0.1 \leq V_{\text {eff }} \leq 0.7$ & $0.1 \leq V_{\text {eff }} \leq 0.7$ \\
$1.33 \leq m_{\mathrm{r}} \leq 1.65$ & $1.33 \leq m_{\mathrm{r}} \leq 1.65$ \\
$10^{-5} \leq m_{\mathrm{i}} \leq 0.5$ & $10^{-5} \leq m_{\mathrm{i}} \leq 0.05$ \\
$\mathrm{Sph}=1.0$ & $0.0 \leq \mathrm{Sph} \leq 1.0$ \\
\hline
\end{tabular}

to describe fine and coarse aerosol modes. The third is set up to represent a cloud: it has wavelength-dependent refractive indices (Segelstein, 1981) and a gamma size distribution with an effective radius of $12.0 \mu \mathrm{m}$ and an effective variance of $0.1 \mu \mathrm{m}$. Since the phase function of a cloud mode is highly peaked, the diffuse intensity field is discretized in 16 streams using the multiple-scattering correction of Nakajima and Tanaka (1988) instead of the eight streams that are used in the RT code of the inversion algorithm.

Partial cloud cover was simulated using the independent pixel approximation (IPA):

$\boldsymbol{I}_{\text {ipa }}=(1-f) \boldsymbol{I}_{\text {clear }}+f \boldsymbol{I}_{\text {cloudy }}$,

where $\boldsymbol{I}$ is the intensity vector that has the Stokes elements as its components and $f$ is the cloud fraction. The vectors $\boldsymbol{I}_{\text {clear }}$ and $\boldsymbol{I}_{\text {cloudy }}$ hold the Stokes elements (at a certain angle and wavelength) for, respectively, a RT model created with only two aerosol modes and a RT model that includes the cloud as a third mode.

The synthetic measurements are created for 400 sets of random aerosol parameters that realistically sample the parameter space for the micro-physical aerosol properties (see Table 2). For 300 of those cases the aerosol optical thickness (AOT) is restricted to low-medium values $\left(0.05 \leq \tau_{550 \mathrm{~nm}}^{\text {fine }} \leq\right.$ 0.35 and $\left.0.05 \leq \tau_{550 \mathrm{~nm}}^{\text {coarse }} \leq 0.35\right)$ as these are expected to be most affected by cloud contamination. Additionally, 50 cases with high fine mode AOT $\left(0.35 \leq \tau_{550 \mathrm{~nm}}^{\text {fine }} \leq 2.0\right.$ and $\left.0.05 \leq \tau_{550 \mathrm{~nm}}^{\text {coars }} \leq 0.35\right)$ and 50 cases with high coarse mode AOT $\left(0.05 \leq \tau_{550 \mathrm{~nm}}^{\text {fine }} \leq 0.35\right.$ and $\left.0.35 \leq \tau_{550 \mathrm{~nm}}^{\text {coarse }} \leq 2.0\right)$ are included.

For every set of aerosol parameters, synthetic observations are created at three solar zenith angles $(\mathrm{SZA}=20,40$, and $60^{\circ}$ ): a clear sky scene and scenes with water cloud fraction ranging from 0.1 to 1.0 by increments of 0.1 for three values of the cloud optical thickness (COT $=1.0,5.0$, and 15.0 at $550 \mathrm{~nm})$.

All the synthetic observations are created using a geometry where the satellite moves in the plane of scattering, observing the aerosol at 15 different angles ranging from 97.0 to $169.0^{\circ}$ for $\mathrm{SZA}=20.0^{\circ}, 77.0$ to $176.0^{\circ}$ for $\mathrm{SZA}=40.0^{\circ}$ and 57.0 to $177.0^{\circ}$ for $\mathrm{SZA}=60.0^{\circ}$. The aerosol is homogeneously distributed over the lowest $2 \mathrm{~km}$ below the cloud which is situated between 2 and $3 \mathrm{~km}$. Lastly, Gaussian noise
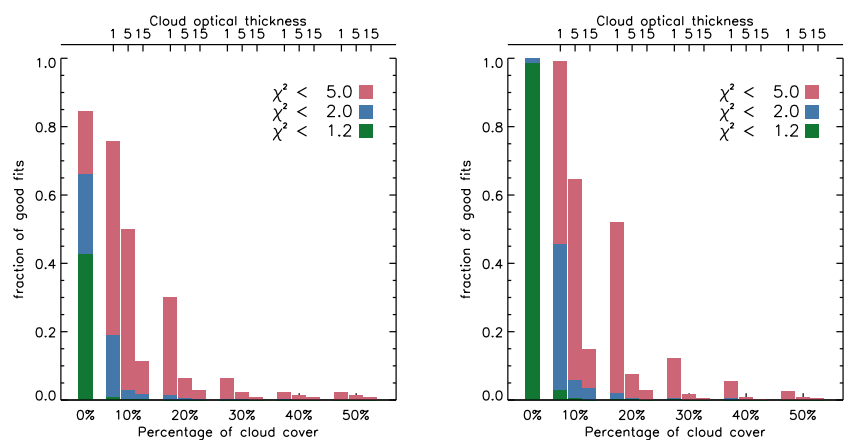

Figure 2. The fraction of good retrievals for three different $\chi^{2}$ filters, per cloud fraction bin, for the normal (left) and perfect first guess retrieval (right). The cloud fraction bins have been further divided into the three optical thicknesses (top axis) that were used to simulate the cloud.

is added to the synthetic measurement, introducing a relative error with a SD of $1.0 \%$ to the intensity and an absolute error of 0.005 to the degree of linear polarization.

\subsection{Sensitivity to liquid cloud contamination}

Two types of retrievals are performed on the data set described in the previous section. One uses a look-up table (consisting of a set of pre-calculated measurements) to obtain a first guess state vector needed in the inversion. Given the non-linear nature of the inversion problem, there is a chance that the retrieval does not converge to the global minimum if the first guess state vector is too far from the true state vector. Therefore, a second type of retrieval is performed which avoids this problem by already starting with the true state vector as the first guess. This retrieval is referred to as the "perfect first guess" retrieval.

Figure 2 shows, for both retrieval types, the fraction of retrievals that fulfil one or more of three different goodnessof-fit criteria at different cloud fractions. While the perfect first guess retrievals obtain a fit with $\chi^{2} \leq 1.2$ in $98 \%$ of the clear sky scenes, only $43 \%$ of the normal retrievals meet this criteria. The reason for a relatively large fraction of these retrievals not to converge to this value of $\chi^{2}$ is that they end up in a local minimum. This happens when the first guess state vector is too far from the true state vector. In principle this can be solved by employing multiple retrievals with a different first guess but this would obviously increase the computational effort significantly. Another approach that can increase the fraction of convergent retrievals is by replacing the look-up table retrieval with a neural network (Di Noia et al., 2014).

It can be seen in Fig. 2 that virtually all cloudy cases are filtered by a strict enough goodness-of-fit criterion $\left(\chi^{2} \leq 1.2\right.$ in this case). The reason for the decrease in the goodness of fit is that the characteristic angular scattering features in DoLP for cloud droplets, such as the cloud bow, which can- 

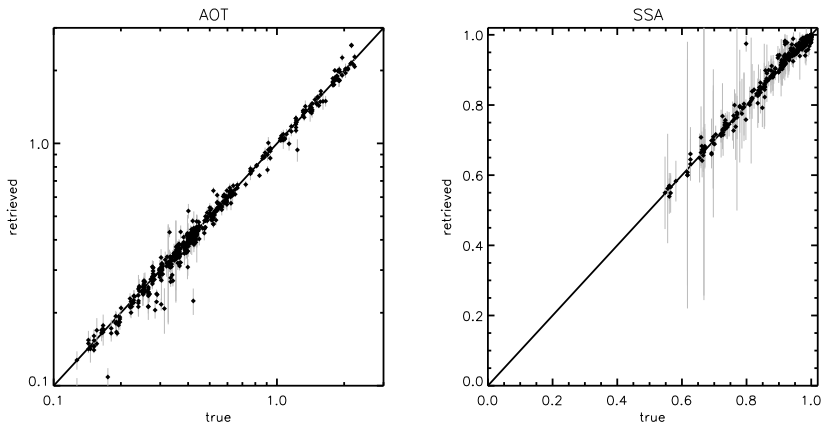

Figure 3. The true vs. retrieved AOT and SSA of those retrievals that pass the $\chi^{2} \leq 1.2$ filter. The error bars indicate the SD (as obtained from the retrieval error covariance matrix) on the retrieved value. The black line shows the $1: 1$ ratio.

not be fitted by the aerosol parameters. With less strict $\chi^{2}$ filters, such as the $\chi^{2} \leq 2.0$ and $\chi^{2} \leq 5.0$ filters, a number of scenes with a small amount of thin cloud cover contribute to the results, which lead to overestimates in the AOT.

The study is repeated for a subset of synthetic measurements with exceptionally large aerosol particles $(3 \leq$ $R_{\text {eff }}^{\text {coarse }} \leq 16$ micron) and the conclusion remains the same (not shown), given that the refractive index of such aerosol particles is significantly different $\left(m_{\mathrm{r}} \geq 1.45\right)$ from the refractive index of water droplets.

Comparisons of the retrieved optical properties and complex refractive indices are shown in Figs. 3 and 4 for those fits with a $\chi^{2} \leq 1.2$. The statistics on the comparison of these and other retrieved parameters are given in Table 3 for three data sets: one where the goodness-of-fit criteria $\left(\chi^{2} \leq\right.$ $1.2)$ has filtered nearly all liquid cloud-contaminated scenes (set 1), one with slightly less strict goodness-of-fit criteria $\left(\chi^{2} \leq 2.0\right)$ that does not filter all liquid cloud-contaminated scenes (set 2) and, lastly, only the liquid cloud-contaminated scenes that meet the $\chi^{2} \leq 2.0$ criteria (set 3 ). The SSA, used in Fig. 3 and Table 3, is additionally filtered on the element of the averaging kernel corresponding to the fine mode imaginary refractive index $\left(A_{m_{\mathrm{i}}^{\text {fine }}} \geq 0.1\right)$ to ensure adequate sensitivity to aerosol absorption. The micro-physical parameters used in Fig. 4 and Table 3 also are additionally filtered by the size of their uncertainty $\left(\sigma_{R_{\text {eff }}^{\text {fine }}} \leq 0.05, \sigma_{R_{\text {eff }}^{\text {coarse }}} \leq 0.1\right.$, $\left.\sigma_{V_{\text {eff }}} \leq 0.1, \sigma_{m_{\mathrm{r}}} \leq 0.04\right)$ or the corresponding element in the averaging kernel $\left(A_{m_{\mathrm{i}}} \geq 0.1\right)$ to ensure that the measurement is sensitive to that particular parameter and thus is constrained by the measurement. It seems from Fig. 3 that the uncertainty in the SSA is somewhat overestimated. This is likely due to the prior error term being chosen too conservatively for one or more parameters.

Figure 3 shows a very good agreement of the true and retrieved optical properties. This is confirmed by the high correlation coefficients and small SDs found in column 1 of Table 3. The cloud-contaminated scenes (set 3 ) consist almost completely of scenes with $10 \%$ thin $(\mathrm{COT}=1.0)$ cloud
Table 3. The correlations, mean and median differences, SDs and retrieval uncertainties are given for the retrieved and derived parameters in three data sets: (i) all retrievals with $\chi^{2} \leq 1.2$, (ii) all retrievals with $\chi^{2} \leq 2.0$ and (iii) only the retrievals in clouded scenes with $\chi^{2} \leq 2.0$. The units for size parameters $R_{\text {eff }}$ and $V_{\text {eff }}$ are in micron.

\begin{tabular}{|c|c|c|c|}
\hline Corr. & $\chi^{2} \leq 1.2$ & $\chi^{2} \leq 2.0$ & $\begin{array}{r}\chi^{2} \leq 2.0 \\
\text { clouds } \\
\text { only }\end{array}$ \\
\hline $\mathrm{AOT}_{670 \mathrm{~nm}}$ & 1.00 & 0.98 & 0.98 \\
\hline $\mathrm{SSA}_{670 \mathrm{~nm}}$ & 0.99 & 0.98 & 0.96 \\
\hline$R_{\text {eff }}^{\text {fine }}$ & 0.91 & 0.85 & 0.78 \\
\hline$V_{\text {eff }}^{\text {fine }}$ & 0.58 & 0.53 & 0.49 \\
\hline $\begin{array}{l}\text { ent } \\
m_{\mathrm{r}}^{\text {fine }}\end{array}$ & 0.92 & 0.87 & 0.80 \\
\hline$m_{\mathrm{i}}^{\text {fine }}$ & 0.90 & 0.83 & 0.86 \\
\hline$R_{\text {eff }}^{1}$ & 0.89 & 0.79 & 0.74 \\
\hline $\begin{array}{l}\text { eff } \\
V_{\text {eff }}^{\text {coarse }}\end{array}$ & 0.85 & 0.76 & 0.71 \\
\hline$m_{\mathrm{r}}^{\text {coarse }}$ & 0.97 & 0.85 & 0.69 \\
\hline$m_{\mathrm{i}}^{\text {coarse }}$ & 0.82 & 0.69 & 0.47 \\
\hline $\mathrm{Sph}_{\text {coarse }}$ & 0.90 & 0.74 & 0.67 \\
\hline \multicolumn{4}{|l|}{ Mean diff. } \\
\hline $\mathrm{AOT}_{670 \mathrm{~nm}}$ & -0.00 & 0.04 & 0.16 \\
\hline $\mathrm{SSA}_{670 \mathrm{~nm}}$ & 0.00 & 0.00 & 0.00 \\
\hline$R_{\text {eff }}^{\text {fine }}$ & -0.00 & -0.00 & -0.01 \\
\hline$V_{\text {eff }}^{\text {tif }}$ & -0.13 & -0.14 & -0.11 \\
\hline$m_{\mathrm{r}}^{\text {fine }}$ & -0.013 & -0.021 & -0.039 \\
\hline$m_{\mathrm{i}}^{\text {fine }}$ & 0.0068 & 0.0091 & 0.0102 \\
\hline$R_{\text {eff }}^{1}$ coarse & -0.09 & -0.12 & -0.06 \\
\hline $\begin{array}{l}\text { ell } \\
V_{\text {eff }}^{\text {coarse }}\end{array}$ & -0.01 & -0.03 & -0.04 \\
\hline$m_{\mathrm{r}}^{\text {coarse }}$ & -0.001 & -0.009 & -0.021 \\
\hline$m_{\mathrm{i}}^{\mathrm{r}}$ coarse & -0.0008 & -0.0011 & -0.0009 \\
\hline $\mathrm{Sph}_{\text {coarse }}$ & 0.03 & 0.03 & 0.00 \\
\hline \multicolumn{4}{|l|}{ Med. diff. } \\
\hline $\mathrm{AOT}_{670 \mathrm{~nm}}$ & -0.00 & 0.00 & 0.09 \\
\hline $\mathrm{SSA}_{670}$ & 0.00 & 0.00 & -0.00 \\
\hline$R_{\text {effe }}^{\text {fine }}$ & 0.01 & 0.01 & 0.00 \\
\hline$V_{\text {eff }}^{\text {tit }}$ & -0.11 & -0.13 & -0.10 \\
\hline $\begin{array}{l}\text { en } \\
m_{\mathrm{r}}^{\text {fine }}\end{array}$ & -0.007 & -0.012 & -0.027 \\
\hline$m_{\mathrm{i}}^{\text {fine }}$ & 0.0013 & 0.0013 & 0.0009 \\
\hline$R_{\text {eff }}^{\text {coarse }}$ & -0.02 & -0.03 & 0.01 \\
\hline $\begin{array}{l}\text { eff } \\
V_{\text {eff }}^{\text {coarse }}\end{array}$ & 0.00 & -0.00 & -0.00 \\
\hline $\begin{array}{l}\text { eff } \\
m_{\mathrm{r}}^{\text {coarse }}\end{array}$ & -0.001 & -0.001 & -0.001 \\
\hline$m_{\mathrm{i}}^{\text {coarse }}$ & 0.0001 & 0.0001 & 0.0000 \\
\hline $\mathrm{Sph}_{\text {coarse }}$ & 0.02 & 0.01 & -0.02 \\
\hline \multicolumn{4}{|l|}{ SD } \\
\hline $\mathrm{AOT}_{670 \mathrm{~nm}}$ & 0.05 & 0.13 & 0.19 \\
\hline $\mathrm{SSA}_{670 \mathrm{~nm}}$ & 0.02 & 0.02 & 0.02 \\
\hline$R_{\mathrm{eff}}^{\text {fine }}$ & 0.05 & 0.07 & 0.08 \\
\hline$V_{\text {eff }}^{\text {fine }}$ & 0.13 & 0.15 & 0.16 \\
\hline$m_{\mathrm{r}}^{\text {fine }}$ & 0.038 & 0.048 & 0.058 \\
\hline$m_{\mathrm{j}}^{\text {fine }}$ & 0.0447 & 0.0530 & 0.0384 \\
\hline$R_{\text {eff }}^{1}$ coarse & 0.29 & 0.40 & 0.42 \\
\hline $\begin{array}{l}\text { eft } \\
V_{\text {eff }}^{\text {coarse }}\end{array}$ & 0.09 & 0.11 & 0.13 \\
\hline $\begin{array}{l}\text { eff } \\
m_{\mathrm{r}}^{\text {coarse }}\end{array}$ & 0.023 & 0.050 & 0.075 \\
\hline$m_{\mathrm{i}}^{\text {coarse }}$ & 0.0043 & 0.0053 & 0.0031 \\
\hline $\mathrm{Sph}_{\text {coarse }}$ & 0.13 & 0.21 & 0.23 \\
\hline \multicolumn{4}{|l|}{ Retr. unc. } \\
\hline $\mathrm{AOT}_{670 \mathrm{~nm}}$ & 0.03 & 0.05 & 0.06 \\
\hline $\mathrm{SSA}_{670 \mathrm{~nm}}$ & 0.05 & 0.07 & 0.04 \\
\hline$R_{\text {effe }}^{\text {fine }}$ & 0.02 & 0.02 & 0.02 \\
\hline $\begin{array}{l}K_{\text {eff }} \\
V_{\text {effe }}^{\text {tine }}\end{array}$ & 0.06 & 0.06 & 0.06 \\
\hline 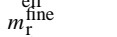 & 0.022 & 0.022 & 0.020 \\
\hline$m_{\mathrm{j}}^{\mathrm{T}}$ & 0.0418 & 0.0553 & 0.0289 \\
\hline $\begin{array}{l}m_{\mathrm{i}} \\
R_{\text {eff }}^{\text {coarse }}\end{array}$ & 0.14 & 0.14 & $\begin{array}{r}0.13 \\
0.13\end{array}$ \\
\hline $\begin{array}{l}\text { eft } \\
V_{\text {eff }}^{\text {coarse }}\end{array}$ & 0.05 & 0.05 & 0.05 \\
\hline$m_{\mathrm{r}}^{\text {coarse }}$ & 0.011 & 0.012 & 0.010 \\
\hline$m_{\mathrm{i}}^{\mathrm{r}}$ & 0.0022 & 0.0019 & 0.0007 \\
\hline $\mathrm{Sph}_{\text {coarse }}$ & 0.09 & 0.10 & 0.07 \\
\hline
\end{tabular}



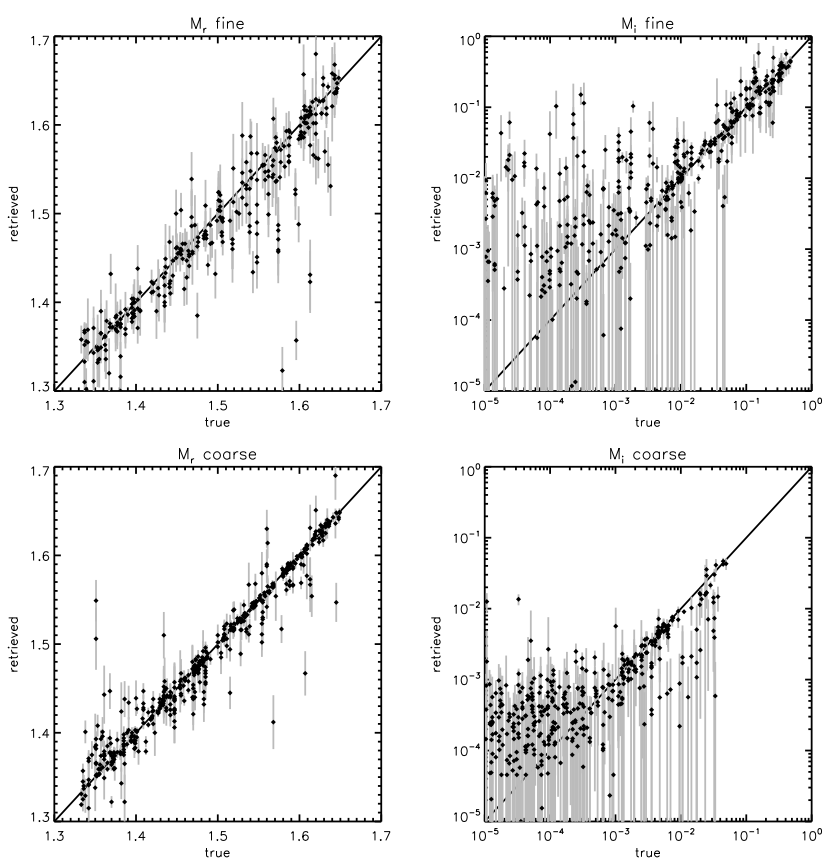

Figure 4. The true vs. retrieved complex refractive indices of those retrievals that pass the $\chi^{2} \leq 1.2$ filter. The error bars indicate the $\mathrm{SD}$ (as obtained from the retrieval error covariance matrix) on the retrieved value. The black line shows the $1: 1$ ratio.

cover. Looking only at the scenes with cloud contamination a bias of 0.16 is observed in the retrieved AOT. This is not reflected in the statistics of set 2, since there are 4 times more clear sky than cloud-contaminated scenes in this data set. For the retrieved SSA, no bias is observed in any of the data sets. There is no significant increase in the retrieval uncertainty of the AOT or SSA due to cloud contamination.

A good agreement is also found for the effective radii of both modes. The effective variance, however, is somewhat harder to retrieve and can be inferred from the lower correlation coefficients. These retrieved parameters are virtually unaffected by cloud contamination; for example, the increase in the SD of the effective radii, from set 1 to set 2 , is only 0.02 for the fine mode and 0.11 for the coarse mode. Note that this increase is largely, if not completely, due to the less strict $\chi^{2}$ filter, not the cloud contamination. For the effective variance, this increase in the SD is small: 0.02 for both modes.

High correlation coefficients are also found for the real refractive index (RRI) of both modes. The mean and median differences in the comparison are small compared to the (mean) retrieval uncertainties. Unexpectedly, a small bias towards underestimation $(\sim 0.04)$ is observed for the $m_{\mathrm{r}}^{\text {fine }}$ in the cloud-contaminated scenes.

The statistics of the imaginary refractive indices are more difficult to interpret due to the large range of values these can assume. As can be seen in Fig. 4, there is not enough information content to retrieve the exact imaginary refractive index when there is very little absorption $\left(m_{\mathrm{i}} \leq 10^{-3}\right)$, corre- sponding to cases with SSA $>0.99$. There is good sensitivity to the imaginary refractive index when absorbing aerosol is present, especially in the fine mode. Absorbing coarse mode aerosol, however, is sometimes underestimated in the retrieval.

The comparison of the true and retrieved coarse mode sphericity also shows a good correlation and no significant bias in all three data sets. In summary, good agreement is found for the AOT, SSA, effective radii, RRIs, $m_{\mathrm{i}}^{\text {fine }}$ and $\mathrm{Sph}_{\text {coarse }}$ in all three data sets. For retrievals in cloudcontaminated scenes a significant bias in the retrieved AOT is expected and an underestimate in $m_{\mathrm{r}}^{\text {fine }}$ might be observed, but no trends are expected in the other retrieved microphysical parameters.

\section{Sensitivity of POLDER-3/PARASOL aerosol retrievals to cloud contamination}

Retrievals of the aerosol and ocean parameters are performed for all the FR and MR PARASOL observations of the year 2006 that are over an ocean surface and in the vicinity (distance $\leq 40 \mathrm{~km}$ ) of an AERONET station (see Table 1). In this section, the sensitivity of the PARASOL aerosol retrievals to cloud contamination, as detected by MODIS, are investigated. Secondly, the goodness-of-fit cloud screening of the aerosol retrieval algorithm is compared with more traditional approaches to cloud screening, which are simulated by discarding the retrieval results for which the MODIS loose or strict cloud mask (see Sect. 2.2) do not indicate a clear sky PARASOL ground pixel. The retrieval results for these different cloud screenings are then compared to the AOT and Ångström exponent ( $₫$ E) from the AERONET direct sun product $(\Delta t \leq 1 \mathrm{~h})$ and the micro-physical aerosol properties and single scattering albedo from the AERONET diffuse sky inversion product $(\Delta t \leq 12 \mathrm{~h})$.

In the retrieval algorithm, the aerosol is assumed to be homogeneously distributed over the lowest $2 \mathrm{~km}$ of the atmosphere. The diffuse intensity field is discretized in eight streams using the multiple-scattering correction of Nakajima and Tanaka (1988). The measurement precision, used to calculate the $\chi^{2}$ of the fit, for both the FR and MR pixels is assumed to be $1.0 \%$ for the intensity and 0.005 (absolute) on the DoLP. Here, the same precision for the FR and MR measurements is assumed because the precision is not dominated by photon noise (which would reduce by combining pixels) but rather by pseudo noise such as stray light, errors due to the POLDER measurement principle, forward model errors, etc. Furthermore, scenes that are only observed at five or fewer viewing angles are discarded.

\subsection{Goodness-of-fit filter}

For both the FR and MR retrievals the fraction of fits that meet one or more of the three $\chi^{2}$ criteria are shown per cloud 

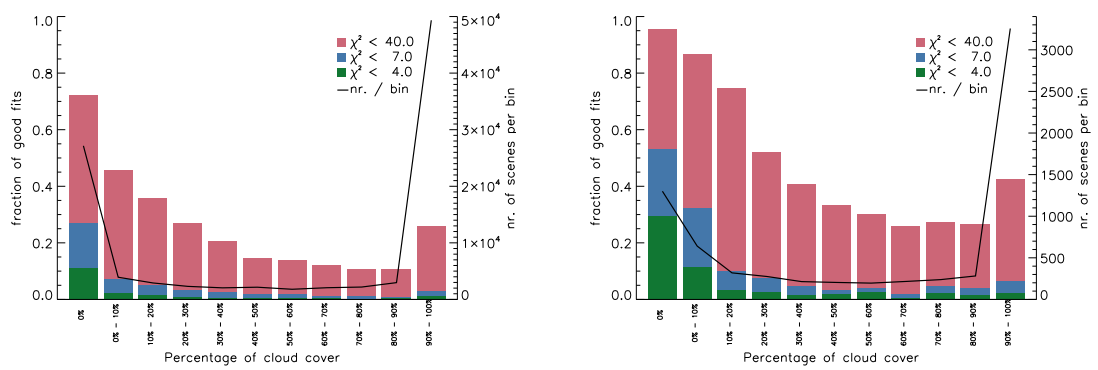

Figure 5. The fraction of good fits per $\chi^{2}$ filter and per cloud fraction bin for full-resolution (left) and medium-resolution (right) PARASOL retrievals. Indicated with a black line are the number of scenes per cloud fraction bin.

fraction bin (using the loose cloud fraction), together with the number of scenes per bin, in Fig. 5. About 27 and 53\% of the retrievals in clear sky scenes pass the $\chi^{2} \leq 7$ filter in the retrievals of, respectively, the FR and MR data sets. At both resolutions, the ability of the aerosol retrieval algorithm to fit the measurements decreases with increasing cloud contamination. In the cloud-contaminated scenes, the fraction of retrievals that converge to $\chi^{2} \leq 7$ is also higher in the MR compared to the FR PARASOL observations. This can be explained by the fact that FR measurements are more sensitive to spatial inhomogeneities caused by e.g., neighboring clouds or land surfaces that are seen in some viewing angles but not in others. An example is given in Fig. 6, which shows a MR measurement and a FR measurement of part of that same scene. The oscillations in the FR measurement are due to clouds. The strong variation in intensity and polarization at the different viewing angles are due to spatial inhomogeneities we refer to as the stereo effect. The MR pixels are (on average) more homogeneous at the different viewing angles. It is, for example, less likely for a cloud to be present in some lines of sight but excluded in others. Therefore, the MR pixels are less affected by the stereo effect. In addition to the sensitivity caused by characteristic scattering features of cloud droplets, this stereo effect introduces an extra sensitivity to cloud contamination in the FR PARASOL observations.

The extent to which a comparison can be made with the fraction of good fits of the synthetic retrievals is limited, since the COT is not known for most of the partially clouded PARASOL pixels at the different cloud fraction bins that are used in Fig. 2. Furthermore, the retrieved $\chi^{2}$ values in PARASOL scenes are expected to be higher on average than those found in the synthetic retrievals. This is in part due to the larger differences between the model and measurements, which are expected because of inhomogeneities in space and time and, to a lesser extent, due to deviations from the assumed vertical distribution and/or size distribution of the aerosol.
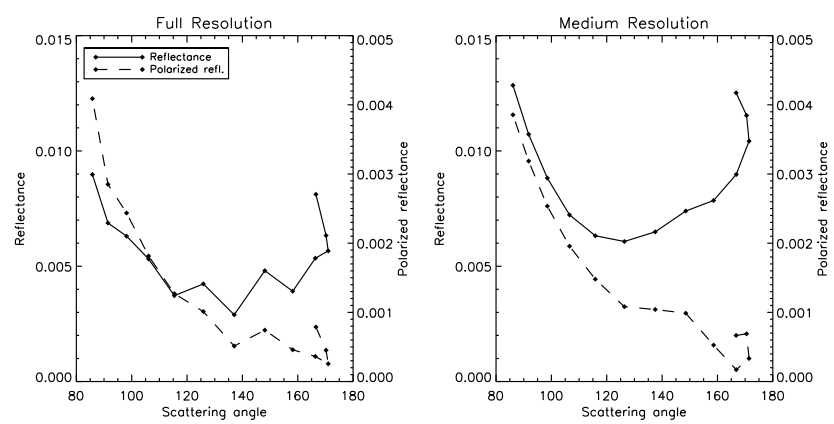

Figure 6. A typical PARASOL observation (in the $865 \mathrm{~nm}$ band) that is affected by stereo effects. While there are no clouds detected by MODIS directly above the full-resolution PARASOL pixel, there are a few clouds roughly $4 \mathrm{~km}$ away. These are included in the medium-resolution PARASOL pixel of which the observation is shown in the panel on the right.

\subsection{Additional filters}

In Fig. 7 the aerosol optical thickness retrieved from the MR PARASOL data is compared to the level 2.0 AERONET observations that are coincident in space (distance $\leq 40 \mathrm{~km}$ ) and time $(\Delta t \leq 1 \mathrm{~h})$. The AERONET AOT is measured in direct sun observations and can be obtained with higher measurement precision than the AOT retrieved from PARASOL observations. It is therefore considered as the "truth" in this comparison. The left panels show the mean AOT bias cumulatively for increasing cloud fraction. This bias is calculated by $\tau^{\mathrm{par}}-\tau^{\mathrm{aer}}$, where $\tau^{\mathrm{par}}$ is retrieved from an individual PARASOL observation and $\tau^{\text {aer }}$ is the mean of all AERONET observations that are within the $1 \mathrm{~h}$ time range. In the panels on the right of Fig. 7, the SDs of the AOT are shown cumulatively for increasing cloud fraction. Since cloud screening has been applied to the AERONET observations, the retrieved AOTs shown in Fig. 7 are subject to some artificial cloud screening. However, there are still numerous occasions where there is a partially clouded PARASOL scene within the $40 \mathrm{~km}$ range, as confirmed by MODIS, while an unobstructed view of the sun is available for the AERONET station within the $1 \mathrm{~h}$ time range. 

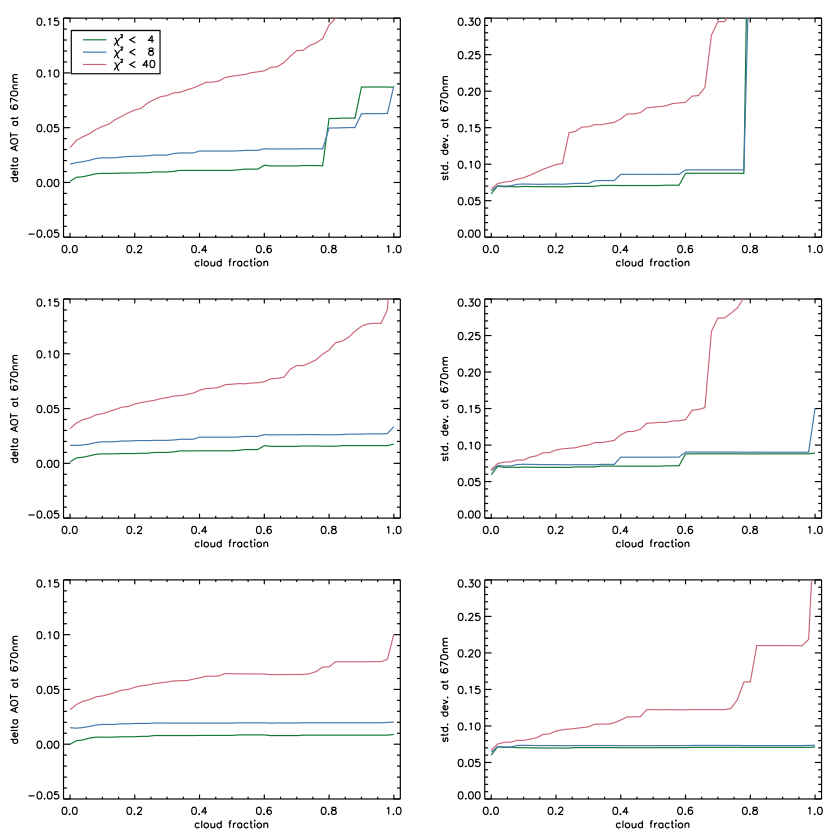

Figure 7. The mean AOT difference between PARASOL and AERONET (left panels) and the SD of the AOT differences between PARASOL and AERONET (right panels) in the $670 \mathrm{~nm}$ band are shown cumulatively for increasing cloud fraction from MODIS, for three different $\chi^{2}$ filters. The results of the top panels have only been filtered with the goodness-of-fit criteria, the middle panels have additionally been filtered on $m_{\mathrm{r}}^{\text {coarse }}>1.335$ and the bottom two panels are filtered on both the $m_{\mathrm{r}}^{\text {coarse }}>1.335$ and on the fraction of co-located MODIS pixels for which the cirrus reflectance flag was set $\left(f_{\text {cirrus }} \leq 10 \%\right)$.

For the $\chi^{2} \leq 40.0$ filter applied in the top panels of Fig. 7, a steady increase in both the AOT bias and SD can be seen with increasing cloud fraction. This indicates that not all cloud-contaminated scenes are filtered by the goodness-offit criteria. In the two stricter $\chi^{2}$ filters, there are a few cloud-contaminated scenes where a decent fit $\left(\chi^{2} \leq 7.0\right)$ is obtained but the AOT is grossly overestimated $(\Delta \tau \geq 5.0)$. This is visible by the jumps in the mean AOT at $f=0.79$, $f=0.89$ and $f=0.99$ for the $\chi^{2} \leq 7.0$ filter. Therefore, apart from the goodness-of-fit filter two additional criteria are needed to discard the cloud-contaminated scenes. One of these criteria deals with water clouds, the other with ice clouds.

In some scenes with partial water cloud cover, the inversion has adjusted the refractive index of the coarse mode to 1.33 (with a sphericity of 1.0). In other words the coarse mode was adjusted to resemble a mode of cloud droplets with optical thicknesses (at $670 \mathrm{~nm}$ ) up to 16. By filtering with the additional criteria $m_{\mathrm{r}}^{\text {coarse }}>1.335$, all these scenes are removed. In total this additional filter removes 109 measurements, compiled from 77 separate AERONET station overpasses by PARASOL, from the MR retrievals that meet the $\chi^{2} \leq 7.0$ criteria. That is less than $2 \%$ of the MR data set.
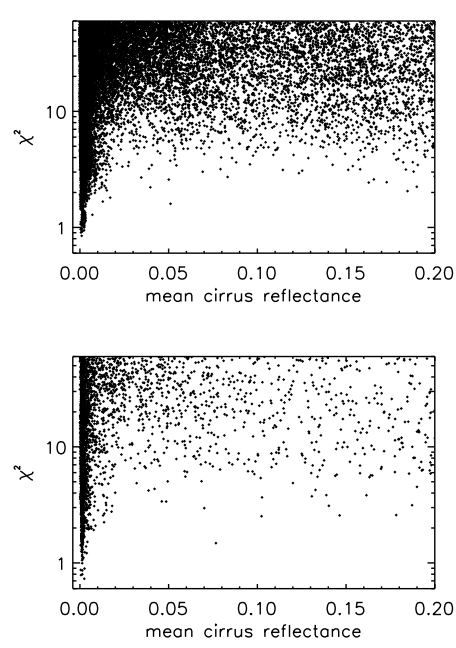

Figure 8. The $\chi^{2}$ of the retrieval vs. the mean cirrus reflectance in the full-resolution (top) and medium-resolution (bottom) scenes. The coarse mode real refractive index filter has been applied to the data in this figure.

The bias and SD obtained with the goodness of fit and coarse mode aerosol refractive index filter are shown in the middle two panels of Fig. 7. Note that the AOT bias and SD are nearly constant with increasing cloud fraction by adding this additional filter criteria. There are, however, still outliers with AOT $>2.8$ at $f=0.38, f=0.60$ and $f=1.00$. These are retrievals in scenes with cirrus clouds.

Even though in most ice cloud scenes no good fits were obtained, which is evident in the sensitivity to the mean cirrus reflection shown in Fig. 8, there are a few overcast ice cloud scenes that do pass the $\chi^{2}$ filter. This leads to a few retrievals of unrealistically large AOTs $\left(\tau_{670 \mathrm{~nm}} \geq 5.0\right)$, and thus a cirrus filter is needed. By requiring that the MODIS cirrus fraction is $\leq 10 \%$ all these scenes are discarded. This removes 250 measurements ( $21 \%$ ) of the MR retrievals that meet the $\chi^{2} \leq 7.0$ criteria. The AOT bias and SD for the results obtained with a goodness-of-fit filter, a coarse mode aerosol refractive index filter and the MODIS cirrus filter, are shown in the bottom two panels of Fig. 7. By applying these two additional filter criteria, the AOT bias for the $\chi^{2} \leq 7.0$ and $\chi^{2} \leq 4.0$ criteria remain constant with increasing cloud fraction. This indicates that there is no significant cloud contamination in these data sets.

The fractions of good fits found at higher cloud fractions are still non-zero after applying these two additional filter criteria. The AOTs retrieved in these scenes do not strongly affect the mean AOT shown in the bottom panels of Fig. 7. The explanation for this is twofold; the fraction of successfully retrieved clear sky scenes outweigh the fraction of successfully retrieved cloudy scenes and no strong overestimate in AOT is retrieved in these latter scenes. This suggests that the successful retrievals in scenes with a high MODIS cloud fraction are falsely identified as cloudy. 

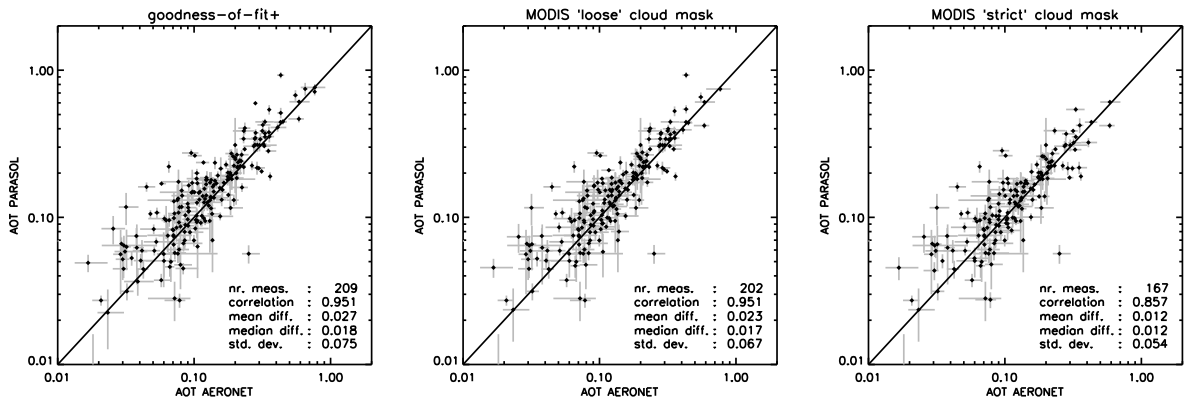

Figure 9. A comparison of the AOTs retrieved from the full-resolution PARASOL observations vs. those retrieved by AERONET (level 2.0) for the three different cloud screenings. The black line shows the $1: 1$ ratio.
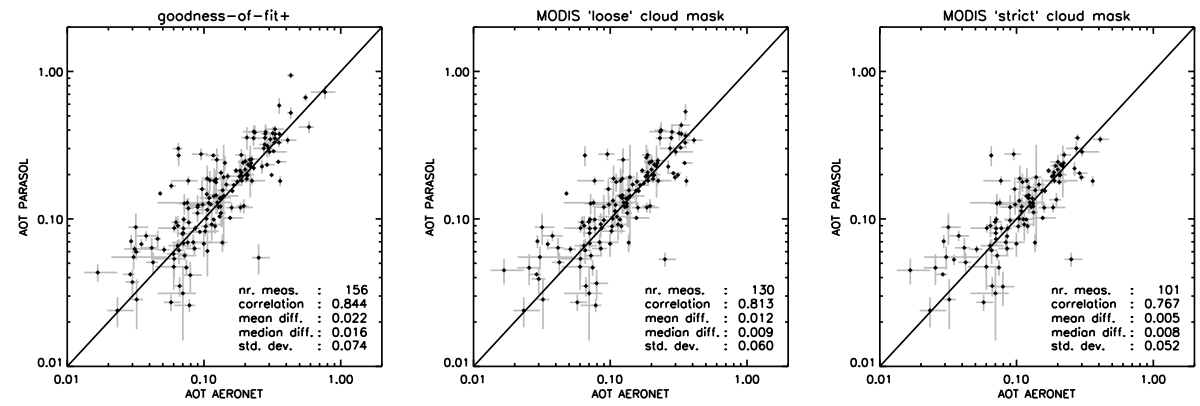

Figure 10. Same as Fig. 9 but for the medium-resolution PARASOL retrievals. The black line shows the $1: 1$ ratio.

Based on the agreement found for the retrieved AOTs, it is concluded that the $\chi^{2} \leq 7.0$ criteria, together with the two additional filters, successfully screen for cloud contamination. Even better agreement, but far less data points (64 and $52 \%$ less for, respectively, the FR and MR retrievals) can be obtained by using the stricter $\chi^{2} \leq 4.0$ criteria. In the remainder of the paper the goodness-of-fit criteria $\chi^{2} \leq 7.0$ is applied. For the sake of brevity, the goodness-of-fit filter together with the coarse mode refractive index filter and cirrus filter will be referred to as the "goodness-of-fit+" filter.

\subsection{Validation of the AOT and Ångström exponent}

In this section the retrieved AOT and $\AA \mathrm{E}$ are compared to those observed with AERONET for three data sets with different cloud screenings: (i) goodness of fit + , (ii) MODIS loose cloud mask and (iii) MODIS strict cloud mask. The results of the latter two cloud screenings are simulated by applying the MODIS loose or strict cloud mask to the PARASOL retrievals. The additional filter criteria on the cirrus fraction is also applied to the two MODIS cloud screenings. The coarse mode refractive index filter is only applied on the goodness-of-fit + filter.

Figures 9 and 10 show the AOT comparisons of the three cloud screenings for, respectively, the FR and MR retrievals. Every data point shows the daily mean AOT (at $670 \mathrm{~nm}$ ) retrieved from PARASOL observations within a range of $40 \mathrm{~km}$ of an AERONET station vs. the mean of the AOTs retrieved from AERONET observations (at $675 \mathrm{~nm}$ ) within $\sim 1 \mathrm{~h}$ of the PARASOL overpass. The grey bars show the range in AOT measurements for AERONET and $1 \sigma$ uncertainty in the retrieval for PARASOL. Some scatter is to be expected due to spatial and temporal inhomogeneity of the atmosphere. Comparisons in the other bands yield almost identical results.

While the MODIS strict cloud mask produces the smallest differences with the AERONET values, it does discard $20 \%$ of the FR and $35 \%$ of the MR results obtained with the goodness-of-fit + filter. Scenes with higher aerosol loading are often flagged cloudy by both MODIS cloud masks, especially at lower resolution, while most of these filtered data points are in good agreement with the optical thicknesses observed by AERONET. The larger absolute errors that can be expected in scenes with higher aerosol loading are one of the causes for the somewhat poorer statistics found in the MR goodness-of-fit + filtered results. When the data points with $\tau^{\mathrm{par}} \geq 0.4$ are excluded (seven data points), the mean and median differences as well as the SD are nearly identical to those found with the loose cloud mask.

The comparison of the ÅEs derived from the AOTs retrieved by PARASOL (using the 490 and $670 \mathrm{~nm}$ bands) and AERONET (using the 500 and $675 \mathrm{~nm}$ bands) is shown in Fig. 11. For this comparison, only data with $\tau_{670 \mathrm{~nm}}^{\mathrm{par}}>0.1$ are included as the information on aerosol size becomes limited for low aerosol loadings. There is a small bias $(-0.12$ to -0.15 ) in the $\AA E$ retrieved by PARASOL and AERONET. 

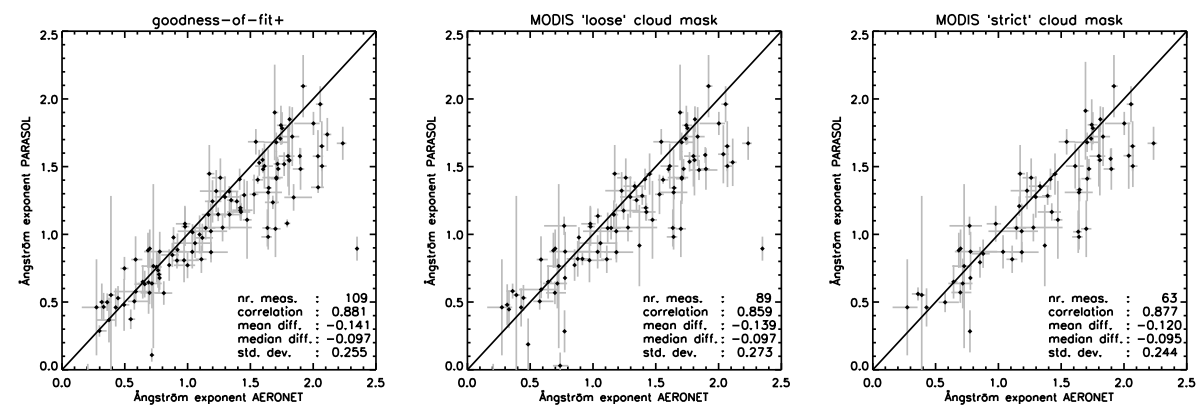

Figure 11. A comparison of the ÅEs retrieved from the medium-resolution PARASOL observations vs. those retrieved by AERONET (level 2.0) for the three different cloud screenings. The black line shows the $1: 1$ ratio.

Note that since this offset is present and of roughly equal strength in all three data sets, it can most likely not be explained in terms of cloud contamination.

\subsection{Validation of the SSA and RRI}

A direct comparison of the micro-physical properties is complicated because of differences in the retrieval methods of AERONET inversion algorithm and the aerosol retrieval algorithm discussed in this paper. The former retrieves a continuous size distribution and wavelength-dependent complex refractive index and derives, among other properties, the single scattering albedo from diffuse sky measurements (almucantar scans) (Dubovik and King, 2000; Dubovik et al., 2002). The aerosol retrieval algorithm discussed in this paper retrieves a bi-modal, log-normal size distribution with two separate, spectrally neutral, complex refractive indices (one for each aerosol mode). However, the agreement found in the $\AA \mathrm{E}$ (see Fig. 11) gives confidence in the retrieved size parameters. Since the SSA is dependent on the microphysical aerosol properties, a comparison of this derived parameter gives an indication of the quality of those retrieved micro-physical properties. In order to facilitate a comparison with the RRI retrieved with the AERONET inversion code, the RRIs of both the fine and coarse modes are weighted by volume and combined to form $m_{\text {comp }}$ (following Hasekamp et al., 2011):

$m_{\text {comp }}=\frac{V^{\text {fine }} m_{\mathrm{r}}^{\text {fine }}+V^{\text {coarse }} m_{\mathrm{r}}^{\text {coarse }}}{V^{\text {fine }}+V^{\text {coarse }}}$,

where superscripts "fine" and "coarse" denote the mode and $V$ stands for volume. This quantity should to some extend reflect the RRI retrieved with the AERONET inversion algorithm. It should be noted, however, that Eq. (2) is only a rough approximation of the RRI retrieved by AERONET. An alternative to Eq. (2) would be to use an optical thickness weighted mean (Zhai et al., 2013), but for any approximation the issue remains that a true quantitative comparison between AERONET and PARASOL is intrinsically impossible for RRI.
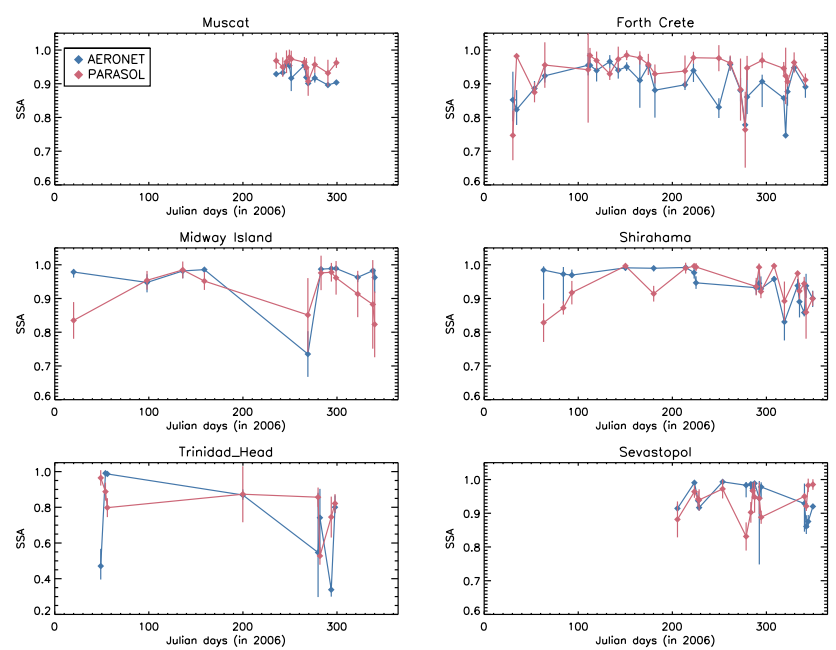

Figure 12. Time series of SSAs (at $670 \mathrm{~nm}$ ) retrieved from the MR PARASOL observations using the goodness-of-fit+ cloud screening vs. those retrieved by AERONET for a number of AERONET stations. The error bars for AERONET show the range of all the observations within $12 \mathrm{~h}$ of the PARASOL overpass and those for PARASOL show the $1 \sigma$ retrieval uncertainty. The MR PARASOL retrievals are additionally filtered on the value of the averaging kernel of the fine mode imaginary refractive index $(\geq 0.1)$ in order to ensure adequate sensitivity to the absorption.

The AERONET diffuse sky measurements are not made as frequently as the direct sun observations from which the AOT product is obtained. Furthermore, the (level 2.0) data obtained from these measurements are subject to strict quality assurance criteria. In particular the criteria that $\tau_{440 \mathrm{~nm}}>0.4$ rejects many observations. In order to get a useful number of coincident observations, comparisons of the SSA and RRI are made with level 1.5 AERONET observations, which have been cloud screened but not quality assured, and the time constraint of the temporal co-location is relaxed to $12 \mathrm{~h}$.

Figure 12 shows time series with the SSAs retrieved from, respectively, PARASOL and AERONET observations at a number of AERONET stations. There is a reasonable agreement in the SSA from the two retrieval approaches. 
Table 4. The statistics for the SSA comparison (at $670 \mathrm{~nm}$ ) at different AERONET stations for the goodness-of-fit+ filtering and, between parentheses, the loose cloud mask filtering. The PARASOL retrievals are additionally filtered on the value of the averaging kernel of the fine mode imaginary refractive index $\geq 0.1$ to ensure adequate sensitivity to the absorption.

\begin{tabular}{lrrrrr}
\hline Station & Nr. obs. & Correlation & Mean diff & Median diff & SD \\
\hline Muscat & $11(8)$ & $0.619(0.531)$ & $0.029(0.029)$ & $0.029(0.035)$ & $0.019(0.019)$ \\
FORTH Crete & $26(21)$ & $0.482(0.479)$ & $0.037(0.049)$ & $0.032(0.039)$ & $0.060(0.056)$ \\
Gosan SNU & $8(5)$ & $0.350(0.263)$ & $0.004(-0.016)$ & $0.009(-0.006)$ & $0.045(0.056)$ \\
Midway Island & $11(9)$ & $0.419(0.432)$ & $-0.036(-0.028)$ & $-0.028(-0.014)$ & $0.074(0.078)$ \\
Shirahama & $18(14)$ & $0.190(0.221)$ & $-0.005(-0.008)$ & $0.006(0.006)$ & $0.063(0.066)$ \\
Trinidad Head & $8(8)$ & $0.001(-0.028)$ & $0.091(0.082)$ & $0.021(0.004)$ & $0.275(0.280)$ \\
Sevastopol & $15(10)$ & $-0.171(-0.452)$ & $-0.012(-0.016)$ & $-0.008(-0.001)$ & $0.066(0.079)$ \\
\hline
\end{tabular}

Table 5. The statistics of the real refractive index comparison (at $670 \mathrm{~nm}$ ) at different AERONET stations for the goodness-of-fit+ filtering and, between parentheses, the loose cloud mask filtering.

\begin{tabular}{lrrrrr}
\hline Station & Nr. obs. & Correlation & Mean diff & Median diff & SD \\
\hline Muscat & $13(8)$ & $0.417(0.308)$ & $-0.075(-0.066)$ & $-0.077(-0.069)$ & $0.037(0.036)$ \\
FORTH Crete & $51(44)$ & $0.253(0.150)$ & $-0.022(-0.017)$ & $-0.021(-0.010)$ & $0.060(0.061)$ \\
Gosan SNU & $11(9)$ & $0.314(0.268)$ & $-0.061(-0.065)$ & $-0.069(-0.069)$ & $0.061(0.056)$ \\
Midway Island & $13(11)$ & $0.232(0.297)$ & $-0.015(-0.036)$ & $-0.021(-0.048)$ & $0.055(0.059)$ \\
Shirahama & $23(18)$ & $-0.100(-0.164)$ & $-0.013(-0.004)$ & $-0.004(-0.002)$ & $0.066(0.068)$ \\
Trinidad Head & $12(13)$ & $0.482(0.618)$ & $-0.088(-0.085)$ & $-0.097(-0.097)$ & $0.063(0.058)$ \\
Sevastopol & $30(22)$ & $0.167(-0.099)$ & $0.001(0.007)$ & $0.007(0.021)$ & $0.049(0.053)$ \\
\hline
\end{tabular}

There are very few differences between the time series obtained with the goodness-of-fit + filtering and the loose cloud mask filtering. The statistics at different AERONET stations and both this filters are listed in Table 4. The mean difference between the AERONET and PARASOL SSA is generally lower than 0.04. Furthermore, the statistics for the MODIS loose mask filtered results are not significantly better than those obtained with the goodness-of-fit + filter.

Figure 13 shows time series of $m_{\text {comp }}$ and the AERONET RRI at $670 \mathrm{~nm}$ for a number of AERONET stations. The statistics of these two values are summarized in Table 5 for those stations where more than five coincident observations are found. It is uncertain what can be expected from this comparison given that $m_{\text {comp }}$ is a crude approximation of the AERONET RRI. For the values retrieved by the aerosol retrieval algorithm, the error bars show the uncertainty in the retrieved value. In general, larger error bars indicate that the retrieval depends strongly on the a priori value for the RRI and associated priori error. For the values retrieved with the AERONET inversion algorithm, the error bars indicate the range of all the measurements that satisfy the time constraint of the temporal co-location. For many cases the error bars overlap, while for others there is a significant difference. Most importantly, the loose cloud mask filtered data set shows fewer data points and nearly identical statistics (see Table 5). In other words, the discrepancies between $m_{\text {comp }}$ and the AERONET RRI cannot be attributed to cloud contamination.
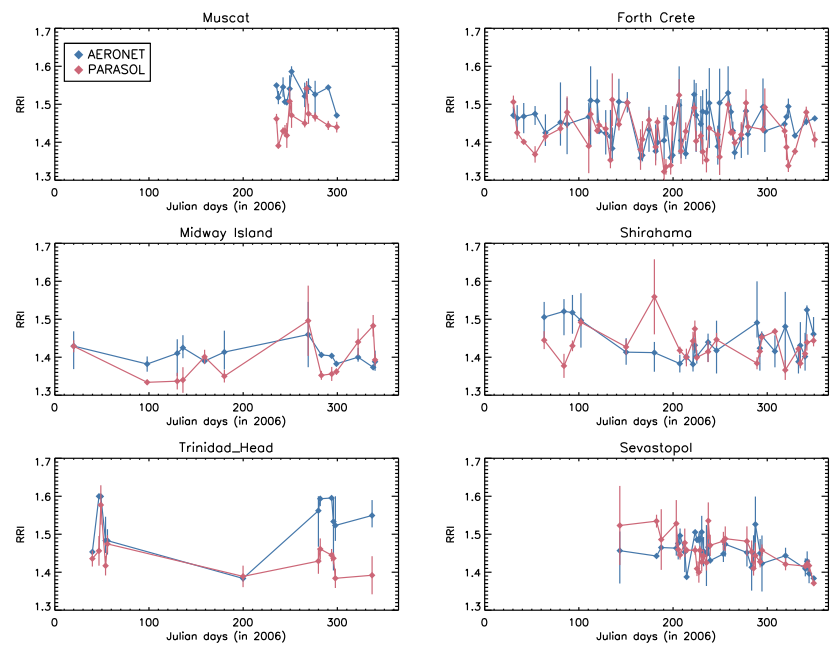

Figure 13. Time series of both the real refractive index (at $670 \mathrm{~nm}$ ) retrieved from AERONET observations and the volume-weighted real refractive index retrieved from the MR PARASOL observations. The goodness-of-fit+ cloud screening is applied on the latter set of observations. The error bars for AERONET show the range of all the observations within $12 \mathrm{~h}$ of the PARASOL overpass and those for PARASOL show the $1 \sigma$ retrieval uncertainty. 


\section{Conclusions}

The effect of cloud contamination on aerosol retrievals from multi-angle photopolarimetric measurements of the POLDER-3 instrument over the ocean is investigated. For retrievals from synthetic measurements it is found that a goodness-of-fit criterion filters out all cases (partially) covered by medium to thick clouds (COT $\geq 5$ ) and virtually all cases with partial thin cloud cover $(\mathrm{COT}=1)$. Aerosol retrievals from PARASOL observations at two spatial resolution, $6 \mathrm{~km} \times 6 \mathrm{~km}(\mathrm{FR})$ and $19 \mathrm{~km} \times 19 \mathrm{~km}(\mathrm{MR})$, are considered. Here, MODIS is used to quantify the cloud contamination for each PARASOL ground pixel. It is found that the FR measurements are more sensitive to cloud contamination than the MR measurements because of so-called stereo effects which occur when, for example, a cloud is seen in one viewing direction but not in the other viewing directions. For MR measurements such effects play a much less important role and the effect of clouds on the measurements is mainly a result of angular scattering features characteristic for cloud droplets. In some cases, the effect of clouds can be described by a coarse aerosol mode with refractive index close to that of water $(\sim 1.33)$. Therefore, an additional criterion which ensures that the coarse mode refractive index is lower than 1.335 is needed. Furthermore, a goodness-of-fit criterion is not always sufficient to filter out ice clouds, which have less distinct angular features than liquid water clouds. To filter out such clouds the MODIS filter based on $1.38 \mu \mathrm{m}$ measurements, or equivalent, is needed. A cloud mask based on a goodness-of-fit criterion, a coarse mode refractive index criterion and a cirrus filter is able to adequately reject cloudy scenes. Moreover, the cloud masks based on MODIS sometimes misinterpret scenes with high aerosol load as cloud contaminated. The aerosol retrievals that pass the goodness of fit, the coarse mode refractive index and cirrus filter do not show a bias and SD with respect to AERONET that is dependent on MODIS cloud fraction. The implication of our findings for future dedicated aerosol polarimeters is that such instruments can fly alone and do not require additional information from a cloud imager. A $1.38 \mu \mathrm{m}$ channel for cirrus detection would be advantageous or even required. Given the large sensitivity of multi-angle photopolarimetric measurements to cloud contamination, a necessary next step is to not use this sensitivity to filter out cloud-contaminated scenes but instead retrieve cloud information simultaneously with aerosol information, describing clouds as an additional size mode with a prescribed refractive index corresponding to water droplets. 


\section{Appendix A}

Table A1. Summary of abbreviations.

\begin{tabular}{ll}
\hline Abbreviation & Meaning \\
\hline ÅE & Ångström exponent \\
AOT & Aerosol optical thickness \\
CCN & Cloud condensation nuclei \\
COT & Cloud optical thickness \\
DoLP & Degree of linear polarization \\
FR & Full resolution \\
IPA & Independent pixel approximation \\
MR & Medium resolution \\
RRI & Real refractive index \\
RT & Radiative transfer \\
SD & Standard deviation \\
SSA & Single scattering albedo \\
SZA & Solar zenith angles \\
\hline
\end{tabular}


Acknowledgements. The authors are very grateful to CNES for providing the POLDER-3/PARASOL data, NASA for providing the MODIS and AERONET data and the AERONET principal investigators and their staff for establishing and maintaining the sites used in this investigation. The authors are also thankful to M. Krijger for providing routines to read and process the MODIS data and P. Tol for sharing his expertise on color schemes for data visualization.

Edited by: A. Kokhanovsky

\section{References}

Ackerman, S. A., Strabala, K. I., Menzel, W. P., Frey, R. A., Moeller, C. C., and Gumley, L. E.: Discriminating clear sky from clouds with MODIS, J. Geophys. Res.-Atmos., 103, 32141, doi:10.1029/1998JD200032, 1998.

Chowdhary, J., Cairns, B., Mishchenko, M. I., Hobbs, P. V., Cota, G. F., Redemann, J., Rutledge, K., Holben, B. N., and Russell, E.: Retrieval of aerosol scattering and absorption properties from photopolarimetric observations over the ocean during the CLAMS experiment, J. Atmos. Sci., 62, 1093-1117, doi:10.1175/JAS3389.1, 2005.

Deschamps, P.-Y., Breon, F.-M., Leroy, M., Podaire, A., Bricaud, A., Buriez, J.-C., and Seze, G.: The POLDER mission: instrument characteristics and scientific objectives, IEEE T. Geosci. Remote, 32, 598-615, doi:10.1109/36.297978, 1994.

Di Noia, A., Hasekamp, O. P., van Harten, G., Rietjens, J. H. H., Smit, J. M., Snik, F., Henzing, J. S., de Boer, J., Keller, C. U., and Volten, H.: Use of neural networks in ground-based aerosol retrievals from multi-angle spectropolarimetric observations, Atmos. Meas. Tech., 7, 9047-9094, doi:10.5194/amtd-7-90472014, 2014.

Dubovik, O. and King, M. D.: A flexible inversion algorithm for retrieval of aerosol optical properties from Sun and sky radiance measurements, J. Geophys. Res.-Atmos., 105, 20673-20696, doi:10.1029/2000JD900282, 2000.

Dubovik, O., Holben, B. N., Lapyonok, T., Sinyuk, A., Mishchenko, M. I., Yang, P., and Slutsker, I.: Non-spherical aerosol retrieval method employing light scattering by spheroids, Geophys. Res. Lett., 29, 1415, doi:10.1029/2001GL014506, 2002.

Dubovik, O., Sinyuk, A., Lapyonok, T., Holben, B. N., Mishchenko, M., Yang, P., Eck, T. F., Volten, H., Muñoz, O., Veihelmann, B., van der Zande, W. J., Leon, J.-F., Sorokin, M., and Slutsker, I.: Application of spheroid models to account for aerosol particle nonsphericity in remote sensing of desert dust, J. Geophys. Res.Atmos., 111, D11208, doi:10.1029/2005JD006619, 2006.

Dubovik, O., Herman, M., Holdak, A., Lapyonok, T., Tanré, D., Deuzé, J. L., Ducos, F., Sinyuk, A., and Lopatin, A.: Statistically optimized inversion algorithm for enhanced retrieval of aerosol properties from spectral multi-angle polarimetric satellite observations, Atmos. Meas. Tech., 4, 975-1018, doi:10.5194/amt-4975-2011, 2011.

Gao, B.-C., Yang, P., Han, W., Li, R.-R., and Wiscombe, W. J.: An algorithm using visible and 1.38- $\mu \mathrm{m}$ channels to retrieve cirrus cloud reflectances from aircraft and satellite data, IEEE T. Geosci. Remote, 40, 1659-1668, doi:10.1109/TGRS.2002.802454, 2002.
Hansen, J. and Travis, L. D.: Light scattering in planetary atmospheres, Space Sci. Rev., 16, 527-610, doi:10.1007/BF00168069, 1974.

Hansen, J., Sato, M., Kharecha, P., and von Schuckmann, K.: Earth's energy imbalance and implications, Atmos. Chem. Phys., 11, 13421-13449, doi:10.5194/acp-11-13421-2011, 2011.

Hasekamp, O. P.: Capability of multi-viewing-angle photopolarimetric measurements for the simultaneous retrieval of aerosol and cloud properties, Atmos. Meas. Tech., 3, 839-851, doi:10.5194/amt-3-839-2010, 2010.

Hasekamp, O. P. and Landgraf, J.: A linearized vector radiative transfer model for atmospheric trace gas retrieval, J. Quant. Spectrosc. Ra., 75, 221-238, doi:10.1016/S0022-4073(01)00247-3, 2002.

Hasekamp, O. P. and Landgraf, J.: Linearization of vector radiative transfer with respect to aerosol properties and its use in satellite remote sensing, J. Geophys. Res.-Atmos., 110, D04203, doi:10.1029/2004JD005260, 2005.

Hasekamp, O. P. and Landgraf, J.: Retrieval of aerosol properties over land surfaces: capabilities of multiple-viewing-angle intensity and polarization measurements, Appl. Optics, 46, 33323344, doi:10.1364/AO.46.003332, 2007.

Hasekamp, O. P., Litvinov, P., and Butz, A.: Aerosol properties over the ocean from PARASOL multiangle photopolarimetric measurements, J. Geophys. Res.-Atmos., 116, D14204, doi:10.1029/2010JD015469, 2011.

Holben, B. N., Tanré, D., Smirnov, A., Eck, T. F., Slutsker, I., Abuhassan, N., Newcomb, W. W., Schafer, J. S., Chatenet, B., Lavenu, F., Kaufman, Y. J., Castle, J. V., Setzer, A., Markham, B., Clark, D., Frouin, R., Halthore, R., Karneli, A., O’Neill, N. T., Pietras, C., Pinker, R. T., Voss, K., and Zibordi, G.: An emerging ground-based aerosol climatology: aerosol optical depth from AERONET, J. Geophys. Res.-Atmos., 106, 12067, doi:10.1029/2001JD900014, 2001.

IPCC: Summary for Policymakers, in: Climate Change 2013: The Physical Science Basis. Contribution of Working Group I to the Fifth Assessment Report of the Intergovernmental Panel on Climate Change, edited by: Stocker, T. F., Qin, D., Plattner, G.-K., Tignor, M., Allen, S. K., Boschung, J., Nauels, A., Xia, Y., Bex, V., and Midgley, P. M., Cambridge University Press, Cambridge, United Kingdom and New York, NY, US, 2013.

Knobelspiesse, K., Cairns, B., Redemann, J., Bergstrom, R. W., and Stohl, A.: Simultaneous retrieval of aerosol and cloud properties during the MILAGRO field campaign, Atmos. Chem. Phys., 11, 6245-6263, doi:10.5194/acp-11-6245-2011, 2011.

Knobelspiesse, K., Cairns, B., Mishchenko, M., Chowdhary, J., Tsigaridis, K., van Diedenhoven, B., Martin, W., Ottaviani, M., and Alexandrov, M.: Analysis of fine-mode aerosol retrieval capabilities by different passive remote sensing instrument designs, Opt. Express, 20, 21457, doi:10.1364/OE.20.021457, 2012.

Kokhanovsky, A. A., Deuzé, J. L., Diner, D. J., Dubovik, O., Ducos, F., Emde, C., Garay, M. J., Grainger, R. G., Heckel, A., Herman, M., Katsev, I. L., Keller, J., Levy, R., North, P. R. J., Prikhach, A. S., Rozanov, V. V., Sayer, A. M., Ota, Y., Tanré, D., Thomas, G. E., and Zege, E. P.: The inter-comparison of major satellite aerosol retrieval algorithms using simulated intensity and polarization characteristics of reflected light, Atmos. Meas. Tech., 3, 909-932, doi:10.5194/amt-3-909-2010, 2010. 
Koren, I., Remer, L. A., Kaufman, Y. J., Rudich, Y., and Martins, J. V.: On the twilight zone between clouds and aerosols, Geophys. Res. Lett., 34, 8805, doi:10.1029/2007GL029253, 2007.

Mishchenko, M. I. and Travis, L. D.: Satellite retrieval of aerosol properties over the ocean using polarization as well as intensity of reflected sunlight, J. Geophys. Res.-Atmos., 102, 1698917013, doi:10.1029/96JD02425, 1997.

Nakajima, T. and Tanaka, M.: Algorithms for radiative intensity calculations in moderately thick atmospheres using a truncation approximation, J. Quant. Spectrosc. Ra., 40, 51-69, doi:10.1016/0022-4073(88)90031-3, 1988.

Ottaviani, M., Knobelspiesse, K., Cairns, B., and Mishchenko, M.: Information content of aerosol retrievals in the sunglint region, Geophys. Res. Lett., 40, 631-634, doi:10.1002/grl.50148, 2013.

Rosenfeld, D.: Aerosols, clouds, and climate, Science, 312, 13231324, doi:10.1126/science.1128972, 2006.

Schuster, G. L., Lin, B., and Dubovik, O.: Remote sensing of aerosol water uptake, Geophys. Res. Lett., 36, 3814, doi:10.1029/2008GL036576, 2009.

Segelstein, D.: The Complex Refractive Index of Water, Department of Physics, University of Missouri-Kansas City, 1981.
Waquet, F., Cairns, B., Knobelspiesse, K., Chowdhary, J., Travis, L. D., Schmid, B., and Mishchenko, M. I.: Polarimetric remote sensing of aerosols over land, J. Geophys. Res.-Atmos., 114, D01206, doi:10.1029/2008JD010619, 2009a.

Waquet, F., Riedi, J., Labonnote, L. C., Goloub, P., Cairns, B., Deuzé, J.-L., and Tanré, D.: Aerosol remote sensing over clouds using a-train observations, J. Atmos. Sci., 66, 2468, doi:10.1175/2009JAS3026.1, 2009b.

Waquet, F., Cornet, C., Deuzé, J.-L., Dubovik, O., Ducos, F., Goloub, P., Herman, M., Lapyonok, T., Labonnote, L. C., Riedi, J., Tanré, D., Thieuleux, F., and Vanbauce, C.: Retrieval of aerosol microphysical and optical properties above liquid clouds from POLDER/PARASOL polarization measurements, Atmos. Meas. Tech., 6, 991-1016, doi:10.5194/amt-6-991-2013, 2013.

Zhai, P.-W., Hu, Y., Hostetler, C. A., Cairns, B., Ferrare, R. A., Knobelspiesse, K. D., Josset, D. B., Trepte, C. R., Lucker, P. L., and Chowdhary, J.: Uncertainty and interpretation of aerosol remote sensing due to vertical inhomogeneity, J. Quant. Spectrosc. Ra., 114, 91-100, doi:10.1016/j.jqsrt.2012.08.006, 2013. 